VOL. $72(2005) \quad[187-196]$

\title{
ASCENDING HNN-EXTENSIONS AND PROPERLY 3-REALISABLE GROUPS
}

\author{
Francisco F. Lasheras
}

\begin{abstract}
In this paper, we show that any ascending HNN-extension of a finitely presented group is properly 3-realisable. We recall that a finitely presented group $G$ is said to be properly 3-realisable if there exists a compact 2-polyhedron $K$ with $\pi_{1}(K) \cong G$ and whose universal cover $\widetilde{K}$ has the proper homotopy type of a (PL) 3-manifold (with boundary).
\end{abstract}

\section{INTRODUCTION}

The most useful constructions in Combinatorial Group Theory are amalgamated free products and HNN-extensions, and they are the two basic examples in the theory of graphs of groups due to Bass and Serre (see [9]). We recall that given a group $G$ and a subgroup $H \leqslant G$ together with monomorphisms (respectively homomorphisms) $\psi, \varphi: H \longrightarrow G$, the group determined by the presentation

$$
\left\langle G, t ; t^{-1} \psi(h) t=\varphi(h), h \in H\right\rangle
$$

is an HNN-extension (respectively pseudo HNN-extension) of $G$ over $H$, with stable letter $t$ (see [12]). In case $H=G$ and $\psi=i d_{G}$, this HNN-extension is called an ascending HNN-extension, and it will be denoted by $G *_{\varphi}$.

We are concerned with the behaviour of the property of being properly 3-realisable (for finitely presented groups) with respect to these constructions. Recall that a finitely presented group $G$ is said to be properly 3-realisable if there exists a compact 2polyhedron $K$ with $\pi_{1}(K) \cong G$ and whose universal cover $\widetilde{K}$ has the proper homotopy type of a p.l. 3-manifold. It is worth mentioning that the property of being properly 3-realisable has implications in the theory of cohomology of groups, in the sense that if $G$ is properly 3-realisable then for some (equivalently any) compact 2-polyhedron $K$ with $\pi_{1}(K) \cong G$ we have $H_{c}^{2}(\widetilde{K} ; Z)$ free Abelian (by manifold duality arguments), and hence so is $H^{2}(G ; \mathbf{Z G})$ (see [10]). It is a long standing conjecture that $H^{2}(G ; \mathbf{Z} G)$ be free Abelian for every finitely presented group $G$. See $[1,6]$ to learn more about properly 3-realisable groups.

Received 28th February, 2005

This work was partially supported by the project MTM 2004-01865.

Copyright Clearance Centre, Inc. Serial-fee code: 0004-9727/05 \$A2.00+0.00. 
In [1] it was shown that amalgamated free products and HNN-extensions of properly 3-realisable groups over a finite cyclic group are properly 3-realisable. The main results of this paper follow.

THEOREM 1.1. Any ascending $H N N$-extension $G *_{\varphi}$ of a finitely presented group $G$ is properly 3-realisable.

Observe that the only property required is that $G$ be finitely presented. More generally, the techniques used in the proof of Theorem 1.1 yield the following.

THEOREM 1.2. Let $G$ be a 1-ended finitely presented group. If the fundamental pro-group of $G$ at infinity is pro-(finitely generated free) and semistable, then $G$ is properly 3-realisable.

CoROLlary 1.3. Let $G$ be an infinite finitely presented group, without torsion in case $G$ has infinitely many ends. If $G$ is simply connected at infinity, then it is properly 3-realisable.

Recall that, given a compact 2-polyhedron $K$ with $\pi_{1}(K) \cong G$ and having $\tilde{K}$ as universal cover, the number of ends of $G$ is the number of ends of $\widetilde{K}$ which equals $0,1,2$ or $\infty$ (see $[9,15]$ ); and $G$ is said to be simply connected at infinity if $\widetilde{K}$ is so, that is, for every compact subpolyhedron $L \subset \widetilde{K}$ there is a compact subpolyhedron $J \supset L$ so that any map $S^{1} \longrightarrow \widetilde{K}-J$ extends to a map $B^{2} \longrightarrow \widetilde{K}-L$.

Note that if $G$ is 1-ended, then any ascending HNN-extension of $G$ is simply connected at infinity [14]. A particularly interesting example is Thompson's group

$$
\begin{aligned}
F & \equiv\left\langle x_{k}, k \geqslant 0 ; x_{i}^{-1} x_{j} x_{i}=x_{j+1}, \text { if } i<j\right\rangle \\
& \cong\left\langle x_{0}, x_{1} ;\left[x_{0} x_{1}^{-1}, x_{0}^{-1} x_{1} x_{0}\right],\left[x_{0} x_{1}^{-1}, x_{0}^{-2} x_{1} x_{0}^{2}\right]\right\rangle
\end{aligned}
$$

whose cohomology modules $H^{*}(F, \mathbf{Z} F$ ) are trivial [3] (and hence $F$ is 1-ended, see [9]); and it is an infinitely iterated HNN-extension. See [4] to learn more about $F$ and similar groups defined by Thompson.

\section{THE MAIN RESULTS}

In this section, we prove Theorems 1.1 and 1.2 and Corollary 1.3.

Proof OF THEOREM 1.1: Let $G$ be a finitely presented group and $\varphi: G \longrightarrow G$ be a monomorphism. It is known, by ([14, Theorem 4.1]), that the corresponding ascending HNN-extension $G *_{\varphi}$ is either 1-ended or 2-ended, depending on whether $G$ is infinite or not. In the second case, we know that $G *_{\varphi}$ is properly 3 -realisable by ([1, Corollary 1.2]). Thus, we may assume that $G$ is infinite and hence $G *_{\varphi}$ is 1 -ended. Note that if $\varphi=i d_{G}$, then $G *_{\varphi}=G \times \mathbf{Z}$ and the conclusion follows from ([6, Theorem 1.1]).

Let $X$ be a compact 2-polyhedron having $\pi_{1}(X) \cong G$, and $\tilde{X}$ as universal cover. Let $f: X \longrightarrow X$ be a cellular map inducing the homomorphism $\varphi$ on the fundamental 
group, and let $\tilde{f}: \widetilde{X} \longrightarrow \widetilde{X}$ be a proper lifting of $f$. Observe that such a map $\tilde{f}$ exists as $f_{*}=\varphi: \pi_{1}(X) \rightarrow \pi_{1}(X)$ is a monomorphism (see [9]). On the other hand, let $Y$ denote the mapping torus of $f$, that is, $Y$ is obtained as a quotient space from the disjoint union $(X \times[0,1]) \cup X$ by identifying $(x, 0)$ with $x$ and $(x, 1)$ with $f(x)$, for all $x \in X$. One can check that $\pi_{1}(Y) \cong G *_{\varphi}$, and it is not hard to see that the universal cover $\tilde{Y}$ of $Y$ can be seen as a collection of copies of the mapping cylinder $M_{\tilde{f}}$ of $\tilde{f}$ attached each other along the copies $q(\tilde{X} \times\{0\}), q(\tilde{X}) \subset M_{\tilde{f}}$ of $\tilde{X}$, where $q:(\tilde{X} \times[0,1]) \sqcup \tilde{X} \rightarrow M_{\tilde{f}}$ is the corresponding quotient map for a generic copy of $M_{\tilde{f}}$ in $\widetilde{Y}$, that is, $(z, 1)$ and $\tilde{f}(z)$ $(z \in \tilde{X})$ get identified via $q$ (see [14]). Moreover, no two copies of $M_{\tilde{f}}$ in $\widetilde{Y}$ intersect along the corresponding subcomplex $q(\tilde{X} \times\{0\})$ of each of them.

We are going to compute the pro-isomorphism type of the fundamental pro-group pro $-\pi_{1}(\tilde{Y})$, see the Appendix. For simplicity, we shall take care of neither base rays nor base points in what follows.

A collection $\widetilde{X}_{a}, \widetilde{X}_{a+1}, \ldots, \widetilde{X}_{a+r}(r \geqslant 1)$ of copies of $\tilde{X}$ in $\widetilde{Y}$ form a chain within $\tilde{Y}$ if for each $0 \leqslant j \leqslant r-1$, there are copies $M_{a, j}$ of $M_{\tilde{f}}$ in $\tilde{Y}$ having $\widetilde{X}_{a+j}, \widetilde{X}_{a+j+1}$ as the corresponding subcomplexes $q(\tilde{X} \times\{0\}), q(\tilde{X}) \subset M_{\tilde{f}}$ respectively. Observe that such a chain is unique from $\tilde{X}_{a}$ to $\tilde{X}_{a+r}$, as $\widetilde{Y}$ is simply connected. On the other hand, we say that two different copies $\tilde{X}_{a}, \widetilde{X}_{b}$ of $\tilde{X}$ in $\tilde{Y}$ are at the same level if there is $r \geqslant 1$ and two chains $\widetilde{X}_{a}, \widetilde{X}_{a+1}, \ldots, \widetilde{X}_{a+r}$ and $\widetilde{X}_{b}, \widetilde{X}_{b+1}, \ldots, \widetilde{X}_{b+r}$ within $\tilde{Y}$ with $\widetilde{X}_{a+r}=\widetilde{X}_{b+r}$. From now on, we shall fix an infinite chain within $\tilde{Y}$, that is, we fix copies $\widetilde{X}_{n}(n \in \mathbf{Z})$ of $\tilde{X}$ in $\tilde{Y}$ so that for each $n \in \mathbf{Z}$, there is a copy $M_{n}$ of $M_{\tilde{f}}$ in $\tilde{Y}$ having $\tilde{X}_{n}, \widetilde{X}_{n+1}$ as the corresponding subcomplexes $q(\tilde{X} \times\{0\}), q(\tilde{X}) \subset \cdot M_{\tilde{f}}$ respectively. Thus, we shall say that a given copy of $\tilde{X}$ in $\tilde{Y}$ is at level $n$ if it is as the same level as $\widetilde{X}_{n} \subset \tilde{Y}$. Let us fix a sequence $C_{1} \subset C_{2} \subset \cdots \subset \tilde{X}$ of compact subcomplexes with $\tilde{X}=\bigcup_{i \geqslant 1} C_{i}$. For any $n \geqslant 1$ and any chain $\gamma$ within $\tilde{Y}$ from a copy of $\widetilde{X}$ at level $-n$ to $\tilde{X}_{n}$, we define a compact subcomplex $D_{n, \gamma} \subset \widetilde{Y}$ as follows. Let

$$
\tilde{X}_{-n, i_{0}}^{(\gamma)}, \tilde{X}_{-n+1, i_{1}}^{(\gamma)}, \ldots, \widetilde{X}_{n-1, i_{2 n-1}}^{(\gamma)}, \widetilde{X}_{n, i_{2 n}}^{(\gamma)}=\widetilde{X}_{n}
$$

be those copies of $\tilde{X}$ which occur in $\gamma$, and denote by $M_{i_{j}, i_{j+1}}^{(\gamma)} \subset \tilde{Y}$ the copy of $M_{\tilde{f}}$ containing $\tilde{X}_{-n+j, i_{j}}^{(\gamma)}$ and $\tilde{X}_{-n+j+1, i_{j+1}}^{(\gamma)}$. Next, using back and forth the cylinder structures, one can build inductively compact subcomplexes

$$
K_{-n+j}^{n} \subset \tilde{X}_{-n+j, i_{j}}^{(\gamma)}(0 \leqslant j \leqslant 2 n)
$$

satisfying:

(i) $K_{-n+j}^{n} \supset C_{n}$ (as subsets of a generic copy of $\tilde{X}$ ), for each $0 \leqslant j \leqslant 2 n$.

(ii) $\tilde{f}^{-1}\left(K_{-n+j+1}^{n}\right)=K_{-n+j}^{n}$, for each $0 \leqslant j \leqslant 2 n$, where $\tilde{f}$ is regarded as a map $\widetilde{X}_{-n+j, i_{j}}^{(\gamma)} \longrightarrow \widetilde{X}_{-n+j+1, i_{j+1}}^{(\gamma)}$ 
(iii) $K_{\alpha}^{m} \supset K_{\beta}^{n}$ (again as subsets of a generic copy of $\tilde{X}$ ) whenever $m \geqslant n$ and for any indexes $-m \leqslant \alpha \leqslant m,-n \leqslant \beta \leqslant n$.

We define

$$
D_{n, \gamma}=\bigcup_{j=0}^{2 n-1} q\left(K_{-n+j}^{n} \times[0,1]\right) \cup K_{-n+j+1}^{n} \subset \tilde{Y}
$$

where

$$
q\left(K_{-n+j}^{n} \times[0,1]\right) \subset M_{i_{j, i}, i}^{(\gamma)},
$$

so that $D_{n, \gamma}$ deformation retracts (using the mapping cylinder structures) onto $K_{n}^{n} \subset \widetilde{X}_{n}$. Furthermore, the closure of $\bigcup_{j=0}^{2 n-1} M_{i_{j}, i_{j+1}}^{(\gamma)}-D_{n, \gamma}$ in $\bigcup_{j=0}^{2 n-1} M_{i_{j}, i_{j+1}}^{(\gamma)}$ deformation retracts onto a (non-compact) subcomplex of $\tilde{X}_{n}-\operatorname{int}\left(K_{n}^{n}\right)$.

Finally, we shall build a particular sequence of compact subcomplexes $D_{1} \subset D_{2}$ $\subset \cdots \subset \tilde{Y}$ with $\tilde{Y}=\bigcup_{i \geqslant 1} D_{1}$, and study the inverse sequence of groups

$$
\{1\} \longleftarrow \pi_{1}\left(\tilde{Y}-\operatorname{int}\left(D_{1}\right)\right) \longleftarrow \pi_{1}\left(\tilde{Y}-\operatorname{int}\left(D_{2}\right)\right) \longleftarrow \cdots
$$

More precisely, we declare $D_{n} \subset \widetilde{Y}$ to be the compact subcomplex $D_{n}=\bigcup_{\gamma} D_{n, \gamma}$, where $\gamma$ ranges over all possible (finitely many) chains within $\tilde{Y}$ from a copy of $\widetilde{X}^{\gamma}$ at level $-n$ to $\widetilde{X}_{n}$. Thus, $D_{n}$ contains all copies of $\widetilde{X}$ in $\widetilde{Y}$ at level $-n$. It is easy to check that the $D_{n}$ 's meet the required properties, by construction. Also, observe that each $D_{n}$ deformation retracts onto $K_{n}^{n} \subset \tilde{X}_{n}$. The picture below roughly describes what $D_{n}$ may look like for $n=1$.

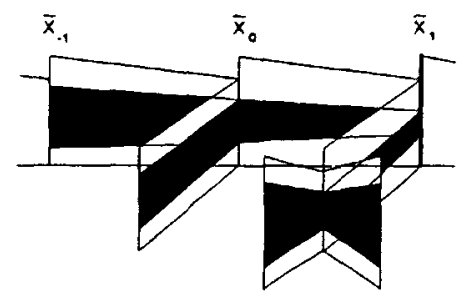

Given $n \geqslant 1$, we define subcomplexes $V_{n}, W_{n} \subset \tilde{Y}$ as follows. We take $V_{n} \subset \tilde{Y}$ to be the union of those copies of $M_{\tilde{f}}$ in $\tilde{Y}$ involved in the construction of $D_{n}$; and $W_{n} \subset \tilde{Y}$ to be the union of those copies $M_{k}$ of $M_{\tilde{f}}$ in $\tilde{Y}$ containing $\tilde{X}_{k}, \tilde{X}_{k+1}$, for all $k \geqslant n$. Note that $V_{n}$ deformation retracts onto $\widetilde{X}_{n}$, and both $V_{n}$ and $W_{n}$ are simply connected. One can check that $\widetilde{Y}-\operatorname{int}\left(D_{n}\right)$ deformation retracts onto

$$
Z_{n}=\left(V_{n}-\operatorname{int}\left(D_{n}\right)\right) \cup W_{n}
$$


Moreover, if $\widetilde{X}_{-n, 1}, \ldots, \widetilde{X}_{-n, j_{n}}$ are all copies of $\widetilde{X}$ in $\widetilde{Y}$ at level $-n$, then

$$
Z_{n}^{\prime}=c l\left(Z_{n}-\bigcup_{r=1}^{j_{n}} \tilde{X}_{-n, r}\right) \subset Z_{n}
$$

deformation retracts onto $W_{n}$ (by construction of the $D_{n}$ 's), and hence $Z_{n}^{\prime}$ is simply connected. Thus, using an argument similar to that in ([6, Theorem 1.1]), one can show that $\pi_{1}\left(\tilde{Y}-\operatorname{int}\left(D_{n}\right)\right) \cong \pi_{1}\left(Z_{n}\right)$ can be expressed as an iterated pseudo HNN-extension starting off from the fundamental group of the (simply connected) complex obtained by gluing each of the copies $\tilde{X}_{-n, 1}, \ldots, \tilde{X}_{-n, j_{n}}$ to $Z_{n}^{\prime}$ along the corresponding copy of a fixed connected component of $\tilde{X}-K_{-n}^{n}$, where each stable letter represents a free generator for $\pi_{1}\left(Z_{n}\right)$. More precisely, if $\tilde{X}-K_{-n}^{n}$ has $l_{n}$ connected components then $\pi_{1}\left(Z_{n}\right)$ is a free group of rank $j_{n}\left(l_{n}-1\right) \geqslant 0$. Furthermore, since $K_{-n}^{n} \subset K_{-n-1}^{n+1}$ (as subsets of a generic copy of $\widetilde{X}$ ) then

$$
\operatorname{rank}\left(\pi_{1}\left(\widetilde{Y}-\operatorname{int}\left(D_{n+1}\right)\right)\right) \geqslant \operatorname{rank}\left(\pi_{1}\left(\widetilde{Y}-\operatorname{int}\left(D_{n}\right)\right)\right) .
$$

Finally, note that the following towers of groups are pro-isomorphic

$$
\begin{gathered}
\{1\} \longleftarrow \pi_{1}\left(\tilde{Y}-\operatorname{int}\left(D_{1}\right)\right) \stackrel{\stackrel{i_{1}}{\longleftarrow}}{\longleftarrow} \pi_{1}\left(\tilde{Y}-\operatorname{int}\left(D_{2}\right)\right) \stackrel{i_{2}}{\longleftarrow} \cdots \\
\{1\} \longleftarrow \operatorname{Im} i_{1} \stackrel{i_{1}}{\longleftarrow} \operatorname{Im} i_{2} \stackrel{i_{2}}{\longleftarrow} \cdots
\end{gathered}
$$

and it is not hard to check that the latter may be regarded as a telescopic tower $\underline{P}$ (see the Appendix), as $\tilde{f}$ maps connected components of $\tilde{X}-K_{-n-1}^{n+1}$ into connected components of

$$
\tilde{X}-K_{-n}^{n+1} \subset \tilde{X}-K_{-n}^{n}(n \geqslant 1),
$$

by construction. Therefore, we conclude that the fundamental pro-group of $\tilde{Y}$ is proisomorphic to a telescopic tower $\underline{P}$.

Next, let $p: \tilde{Y} \longrightarrow Y$ be the covering projection, and pick a base ray $\omega$ in $\widetilde{Y}$. Since $\tilde{Y}$ is 2-dimensional and one-ended, there exist spherical objects $S_{\omega}^{2}$ and $S_{\omega^{\prime}}^{2}$ and a proper homotopy equivalence $\tilde{Y} \vee S_{\omega}^{2} \simeq B(\underline{P}) \vee S_{\omega^{\prime}}^{2}$, by Theorem 3.2. Let $V \subset \widetilde{K}$ be the set of vertices in $\omega([0, \infty))$, with $p(V)=\left\{v_{1}, \ldots, v_{r}\right\} \subset Y$, and denote by $\widehat{Y}$ the polyhedron obtained from $\tilde{Y} \vee S_{\omega}^{2}$ by attaching one sphere $S^{2}$ through every vertex in $p^{-1}(p(V))-\omega([0, \infty))$. Thus, $\widehat{Y}$ is the universal cover of the compact 2-polyhedron obtained from $Y$ by attaching one sphere $S^{2}$ at each of the vertices $v_{1}, \ldots, v_{r}$ (which is homotopy equivalent to a wedge $\left.Y \vee\left(\vee_{i=1}^{r} S^{2}\right)\right)$. On the other hand, $\widehat{Y}$ is proper homotopy equivalent to a polyhedron $Q$ obtained from $B(\underline{P}) \vee S_{\omega^{\prime}}^{2}$ by attaching infinitely many spheres $S^{2}$ in a proper way (that is, via the corresponding proper homotopy equivalence given by Theorem 3.2). Finally, the proper homotopy type of the proper wedge $B(\underline{P}) \vee S_{\omega^{\prime}}^{2}$ can be represented by the closed subpolyhedron in $\mathbf{R}^{3}$ shown in the figure below. It is 
then easy to check that the proper homotopy type of $Q$ can also be represented by a closed subpolyhedron $\widehat{Q}$ in $\mathbf{R}^{3}$.

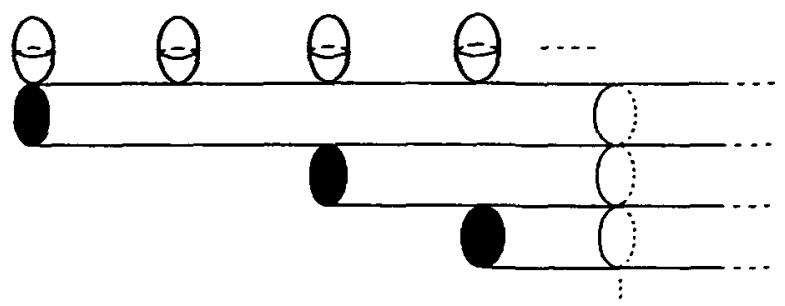

Therefore, the universal cover of the compact 2-polyhedron $Y \vee\left(\vee_{i=1}^{r} S^{2}\right)$ (with $\left.\pi_{1}\left(Y \vee\left(\vee_{i=1}^{r} S^{2}\right)\right) \cong G *_{\varphi}\right)$ turns out to be proper homotopy equivalent to the 3-manifold obtained by taking a regular neighbourhood of $\widehat{Q}$ in $\mathbf{R}^{3}$, and hence $G *_{\varphi}$ is properly 3-realisable.

REMARK 2.1. Note that the argument used in the proof of Theorem 1.1 above gives an alternative proof of the fact that any ascending HNN-extension of a (finitely presented) group is semistable at infinity (see [14]).

Proof OF THEOREM 1.2: Let $X$ be a compact 2-polyhedron with $\pi_{1}(X) \cong G$ and having $\widetilde{X}$ as universal cover, and let $K_{1} \subset K_{2} \subset \cdots \subset \widetilde{X}$ be a sequence of compact subsets so that $\tilde{X}=\bigcup_{i \geqslant 1} K_{i}$. The inverse system

$$
\{1\} \longleftarrow \pi_{1}\left(\tilde{X}-K_{1}\right) \stackrel{i_{1}}{\longleftarrow} \pi_{1}\left(\tilde{X}-K_{2}\right) \stackrel{i_{2}}{\longleftarrow} \cdots
$$

is then pro-isomorphic to a tower $\underline{F}=\left\{F_{0} \stackrel{\phi_{1}}{\longleftarrow} F_{1} \stackrel{\phi_{2}}{\longleftarrow} F_{2} \longleftarrow \cdots\right\}$ of finitely generated free groups which is semistable, by hypothesis. It suffices to show that $E$ is in fact proisomorphic to a telescopic tower $\underline{P}$, and then apply the same argument as in the proof of Theorem 1.1, as $G$ is 1-ended.

For this, recall that $F$ is said to be semistable if for each $k$ there is $n \geqslant k$ so that for every $m \geqslant n$ we have $\operatorname{Im}\left(\phi_{k+1} \cdots \phi_{m}\right)=\operatorname{Im}\left(\phi_{k+1} \cdots \phi_{n}\right) \subset F_{k}$. It is well known that if $\underline{F}$ has this property then it is pro-isomorphic to a tower of groups

$$
\underline{P}=\left\{P_{0} \stackrel{\psi_{1}}{\longleftarrow} P_{1} \stackrel{\psi_{2}}{\longleftarrow} P_{2} \longleftarrow \cdots\right\}
$$

where the bonding maps $\psi_{n}$ are all epimorphisms. Moreover, there is a level morphism $\left\{i_{n}\right\}$ inducing this pro-isomorphism such that every $i_{n}: P_{n} \longrightarrow F_{n}$ is a monomorphism between finitely generated groups (see [13]). Thus, $\underline{P}$ is a tower of finitely generated free groups in which all bonding maps are epimorphisms. Finally, since $\psi_{n}: P_{n} \longrightarrow P_{n-1}$ is an epimorphism between free groups, it follows from [8] that $P_{n}$ is a free product $P_{n}=P_{n}^{\prime} * P_{n}^{\prime \prime}$, where $\psi_{n}\left(P_{n}^{\prime \prime}\right)=\{1\}$ and $\psi_{n} \mid P_{n}^{\prime}$ is an isomorphism. Therefore, each $\psi_{n}$ is a projection and hence $\underline{P}$ can be regarded as a telescopic tower, as each $P_{n}^{\prime \prime}$ is finitely generated by the Grushko-Neumann theorem. 
Proof of Corollary 1.3: Let $G$ be an infinite finitely presented group, and suppose $G$ is simply connected at infinity. We show that $G$ is properly 3-realisable. Of course, if $G$ is 2-ended then it is properly 3-realisable by ([1, Corollary 1.2]). On the other hand, if $G$ has infinitely many ends, then $G$ splits as a non-trivial free product (as $G$ is torsion-free, by hypothesis) by the Stallings' Structure theorem (see [15]), and one can easily check that each of the factors must be simply connected at infinity. Moreover, this splitting process must terminate after finitely many steps, by Dunwoody's accessibility result [7]. Thus, we may as well assume that $G$ is 1-ended and simply connected at infinity, since the free product of properly 3-realisable groups is properly 3-realisable, by $([\mathbf{1}$, Lemma 3.2$])$. For this, note that in this case pro $-\pi_{1}(\widetilde{K})$ is pro-isomorphic to the trivial (telescopic) tower, where $K$ is a compact 2-dimensional $C W$-complex with $\pi_{1}(K) \cong G$ and having $\widetilde{K}$ as universal cover (see [9]). Therefore, using an argument similar to that of Theorem 1.1 (as $\widetilde{K}$ is 1-ended), one shows that $G$ is indeed properly 3-realisable.

\section{ApPEndix}

This section is intended to provide the background and notation needed in the previous section, as well as to indicate what is behind those results. In what follows, we shall be working within the category tow $-G r$ of towers of groups whose objects are inverse sequences of groups

$$
\underline{A}=\left\{A_{0} \stackrel{\phi_{1}}{\longleftarrow} A_{1} \stackrel{\phi_{2}}{\longleftarrow} A_{2} \longleftarrow \cdots\right\}
$$

A morphism in this category will be called a pro-morphism. See $[2,13]$ for a general reference.

A tower $\underline{L}$ is a free tower if it is of the form

$$
\underline{L}=\left\{L_{0} \stackrel{i_{1}}{\longleftarrow} L_{1} \stackrel{i_{2}}{\longleftarrow} L_{2} \longleftarrow \cdots\right\}
$$

where $L_{i}=\left\langle B_{i}\right\rangle$ are free groups of basis $B_{i}$ such that $B_{i+1} \subset B_{i}$, the differences $B_{i}-B_{i+1}$ are finite and $\bigcap_{i=0}^{\infty} B_{i}=\emptyset$, and the bonding homomorphisms $i_{k}$ are given by the corresponding basis inclusions. On the other hand, a tower $\underline{P}$ is a telescopic tower if it is of the form

$$
\underline{P}=\left\{P_{0} \stackrel{p_{1}}{\longleftarrow} P_{1} \stackrel{p_{2}}{\longleftarrow} P_{2} \longleftarrow \cdots\right\}
$$

where $P_{i}=\left\langle D_{i}\right\rangle$ are free groups of basis $D_{i}$ such that $D_{i-1} \subset D_{i}$, the differences $D_{i}-D_{i-1}$ are finite (possibly empty), and the bonding homomorphisms $p_{k}$ are the obvious projections.

We shall also use the full subcategory $(G r$, tow $-G r)$ of Mor(tow $-G r)$ whose objects are arrows $\underline{A} \longrightarrow G$, where $\underline{A}$ is an object in tow $-G r$ and $G$ is a group regarded as a constant tower whose bonding maps are the identity. Morphisms in $(G r$, tow $-G r)$ will also be called pro-morphisms. 
From now on, $X$ will be a (strongly) locally finite CW-complex. A proper map $\omega:[0, \infty) \longrightarrow X$ is called a proper ray in $X$. We say that two proper rays $\omega, \omega^{\prime}$ define the same end if their restrictions $\left.\omega\right|_{\mathbf{N}},\left.\omega\right|_{\mathbf{N}}$ are properly homotopic. Moreover, we say that they define the same strong end if $\omega$ and $\omega^{\prime}$ are in fact properly homotopic.

Given a base ray $\omega$ in $X$ and a collection of compact subsets $C_{1} \subset C_{2} \subset \cdots \subset X$ so that $X=\bigcup_{n=1}^{\infty} C_{n}$, the following tower

$$
\text { pro }-\pi_{1}(X, \omega)=\left\{\pi_{1}(X, \omega(0)) \leftarrow \pi_{1}\left(X-C_{1}, \omega\left(t_{1}\right)\right) \leftarrow \pi_{1}\left(X-C_{2}, \omega\left(t_{2}\right)\right) \leftarrow \cdots\right\}
$$

can be regarded as an object in $(G r$, tow $-G r)$ and it is called the fundamental pro-group of $(X, \omega)$, where $\omega\left(\left[t_{i}, \infty\right)\right) \subset X-C_{i}$ and the bonding homomorphisms are induced by the inclusions. This tower does not depend (up to pro-isomorphism) on the sequence of subsets $\left\{C_{i}\right\}_{i}$. It is worth mentioning that if $\omega$ and $\omega^{\prime}$ define the same strong end, then pro $-\pi_{1}(X, \omega)$ and pro $-\pi_{1}\left(X, \omega^{\prime}\right)$ are pro-isomorphic. In particular, we may always assume that $\omega$ is a cellular map. Moreover, if $X$ is strongly connected at each end (that is, any two proper rays defining the same end define the same strong end), then $\pi_{1}^{e}(X, \omega)=\lim _{-}$pro $-\pi_{1}(X, \omega)$ is a well-defined useful invariant which only depends (up to isomorphism) on the end determined by $\omega$ (see [11]). In a similar way, one can define objects in $(G r$, tow $-G r)$ corresponding to the higher homotopy pro-groups of $(X, \omega)$.

Definition 3.1: Given $n \geqslant 1$, a tree $T$ and a proper ray $\omega:[0, \infty) \longrightarrow T$, a spherical object $S_{\omega}^{n}$ under $T$ is a space obtained from $T$ by attaching finitely $n$-spheres $S^{n}$ at each vertex of $\omega([0, \infty))$. Observe that any two of such spherical objects (along $\omega$ ) are proper homotopy equivalent (under $T$ ), by ([2, Proposition 4.5(b)]).

The following result, which can be thought of as a special case of a proper version of Whitehead's theorem for compact 2-dimensional $C W$-complexes, will be crucial for the proof of those results in Section 2.

THEOREM 3.2. ([5, Corollary 6.4]) If $X$ is a one-ended 2-dimensional locally finite $C W$-complex, then the following are equivalent

(a) pro $-\pi_{1}(X, \omega)$ is pro-isomorphic to a (coproduct) tower of the form $\underline{L} \vee \underline{P}$.

(b) There exist spherical objects $S_{\omega}^{2}$ and $S_{\omega^{\prime}}^{2}$ and a proper homotopy equivalence (under $[0, \infty)) X \vee S_{\omega}^{2} \simeq B(\underline{L} \vee \underline{P}) \vee S_{\omega^{\prime}}^{2}$.

Here, $\left(B(\underline{L} \vee \underline{P}), \omega^{\prime}\right)$ is the properly based 2-polyhedron defined as the proper wedge (that is, along a base ray) of a one-ended spherical object $S_{\varepsilon}^{1}$, with pro $-\pi_{1}\left(S_{\varepsilon}^{1}, \omega^{\prime}\right) \cong \underline{L}$ $\left(\omega^{\prime}:[0, \infty) \hookrightarrow S_{\varepsilon}^{1}\right.$ the canonical inclusion), and a proper wedge $C$ of a decreasing sequence (possibly infinite) of cylinders $C_{n}=S^{1} \times[n, \infty)$ and/or Euclidean planes

$$
\mathbf{R}_{m}^{2}=S^{1} \times[m, \infty) / S^{1} \times\{m\}
$$

attached along the half line $[0, \infty)$ for which pro $-\pi_{1}\left(C, \omega^{\prime}\right) \cong \underline{P}$, with $\omega^{\prime}:[0, \infty) \hookrightarrow C$ the canonical inclusion. Thus, $B(\underline{L} \vee \underline{P})$ can be seen as a "proper Eilenberg-MacLane space" 
$K(\underline{L} \vee \underline{P}, 1)$ and its proper homotopy type can be represented by a closed subpolyhedron in $\mathbf{R}^{3}$ of the type as shown in the figure below.

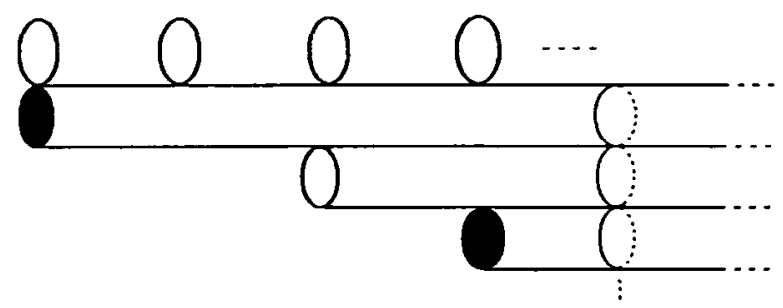

\section{REFERENCES}

[1] R. Ayala, M. Cárdenas, F.F. Lasheras and A. Quintero, 'Properly 3-realizable groups', Proc. Amer. Math. Soc. 133 (2005), 1527-1535.

[2] H-J. Baues and A. Quintero, Infinite homotopy theory, K-monographs in Mathematics 6 (Kluwer Academic Publishers, Dordrecht, 2001).

[3] R. Brown and R. Geoghegan, 'An infinite-dimensional torsion-free $F P_{\infty}$ group', Invent. Math. 77 (1984), 367-381.

[4] J.W. Cannon, W.J. Floyd and W.R. Parry, 'Introductory notes on Richard Thompson's groups', Enseig. Math. 42 (1996), 215-256.

[5] M. Cárdenas, F.F. Lasheras, F. Muro and A. Quintero, 'Proper L-S category, fundamental pro-groups and 2-dimensional proper co- $H$-spaces', Topology Appl. (to appear).

[6] M. Cárdenas, F.F. Lasheras and R. Roy, 'Direct products and properly 3-realisable groups', Bull. Austral. Math. Soc. 70 (2004), 199-206.

[7] M.J. Dunwoody, 'The accessibility of finitely presented groups', Invent. Math. 81 (1985), 449-457.

[8] H. Federer and B. Jonsson, 'Some properties of free groups', Trans. Amer. Math. Soc. 68 (1950), 1-27.

[9] R. Geoghegan, Topological methods in group theory, (book in preparation).

[10] R. Geoghegan and M. Mihalik, 'Free abelian cohomology of groups and ends of universal covers', J. Pure Appl. Algebra 36 (1985), 123-137.

[11] - R. Geoghegan and M. Mihalik, 'The fundamental group at infinity', Topology 35 (1996), 655-669.

[12] R.C. Lyndon and P.E. Schupp, Combinatorial group theory (Berlin, Heidelberg, New York, 1977).

[13] S. Mardešic and J. Segal, Shape theory (North-Holland, Amsterdam, New York, 1982).

[14] M. Mihalik, 'Ends of groups with the integers as quotient', J. Pure Appl. Algebra 35 (1985), 305-320.

[15] P. Scott and C.T.C. Wall, 'Topological methods in group theory', in Homological group theory, London Math. Soc. Lecture 36 Notes (Cambridge Univ. Press, Cambridge, 1979), pp. 137-204. 
Departamento de Geometría y Topología

Universidad de Sevilla

Apdo 1160, 41080-Sevilla

Spain

e-mail: lasheras@us.es 